\title{
Procesos de enseñanza y aprendizaje significativo asociados a la ingeniería industrial, uso de la lúdica, el modelamiento y la simulación en el laboratorio integral de ingeniería industrial en Unicatólica (lúdicas lab3i)*
}

Significant teaching and learning processes associated with industrial engineering, use of ludic, modeling and simulation in the integral laboratory of industrial engineering in Unicatólica (lúdicas lab3i)

\section{Resumen}

El presente trabajo corresponde al desarrollo de un proyecto de investigación el cual se encuentra en curso y cuya finalidad se enfoca en el establecimiento de los criterios, así como la línea base para coordinar las estrategias pedagógicas y didácticas de la Facultad de Ciencias Básicas e Ingeniería de Unicatólica, en especial aquellas que se asocian a los procesos de enseñanza y aprendizaje propios del quehacer de la Ingeniería Industrial. En tal sentido, se consideran los elementos que emergen del aprendizaje basado en problemas (ABP) y el aprendizaje significativo con una panorámica orientada a la lúdica, el modelamiento y la simulación de situaciones que se 
materializan en el devenir de la disciplina de Ingeniería Industrial. De otra, parte se hace referencia al proceso evolutivo de la formación universitaria en ingeniería, la cual ha tenido un enfoque que se adentra en el sustento teórico y conceptual en el que se privilegia la memorización y mecanización de los procesos. Este hecho dificulta la comprensión de los fenómenos, así como su modelización con ejemplos adaptados a los contextos industriales.

Particularmente en Colombia este hecho generó una amplificación de la brecha de atraso tecnológico en los procesos asociados con el sistema productivo. De acuerdo con lo anterior, en este artículo se consideran algunas de las tendencias que se requieren al respecto a nivel global y dentro del contexto nacional para la formación integral de ingenieros industriales, capaces de hacerle frente al posicionamiento competitivo del país, con una clara dimensión de la toma de decisiones sustentables y responsables con la sociedad.

Palabras clave: procesos de enseñanza y aprendizaje significativo, ABP, lúdica, modelamiento, simulación.

\section{Abstract}

The present work corresponds to the development of a research project which is ongoing. Its purpose focuses on the establishment of criteria, as well as a baseline to coordinate pedagogical and didactic strategies for the Faculty of Basic Sciences and Engineering of Unicatólica, especially those associated with the teaching and learning processes of Industrial Engineering. In this sense, elements that emerge from trouble-based learning (TBL) and meaningful learning with a scenic focus oriented to ludic, modeling and simulation of situations that materialize in the discipline of Industrial Engineering are considered. On the other hand, part refers to the evolutionary process of university engineering training. It has had an approach that delves into theoretical and conceptual sustenance where memorization and mechanization of processes was preferred. Such fact makes it difficult to understand phenomena behavior, as well as their modeling with adapted samples of the industrial context.

Particularly in Colombia this fact takes to an amplification of the late pad in the technological process inside productive systems. According to the above sentences, this paper considers trends at a global level and national context required for the integrated teaching of engineers. This to be able to respond to competitive country performance with a clair dimension to take sustainable and socially responsible decisions.

Keywords: significant teaching and learning processes, TBL, playful, modeling, simulation.

\section{Introducción}

La configuración actual de la educación en términos generales, así como el rápido avance de los sistemas económicos hacen necesario el desarrollo de procesos de enseñanza y aprendizaje que conduzcan a la construcción de estructuras robustas de conocimiento con significancia para la disciplina de la ingeniería. Esto es posible si se logra dirigir la atención a las problemáticas y necesidades presentes en los contextos local, regional y nacional en alineación con las estrategias pedagógicas y didácticas asociadas a la enseñanza de la Ingeniería Industrial, dado que la prioridad de atención está en los criterios o principios que les permiten a estas consolidar un aprendizaje significativo. 
En virtud de ello, la acción de generar conocimiento supone compartir experiencias y fortalecer la dinámica colaborativa de los ambientes surgidos del aprendizaje basado en problemas (ABP) (Escribano González y Valle López, 2018).

En ese sentido, el uso de la tecnología complementada con la innovación hace que los vertiginosos cambios en las dinámicas de los sectores industriales requieran de profesionales cuyas competencias les permitan tomar decisiones sustentadas en alternativas, escenarios y métodos que sean objetivamente claros. Por ello, el uso del modelamiento permite la representación de contextos específicos contrastados con la realidad de los sistemas socioeconómicos, empresariales y universitarios. La construcción de modelos permite a los estudiantes de ingeniería y en especial a los de Ingeniería Industrial, forjar un pensamiento crítico. La principal ventaja es que ofrece una visión con enfoque creativo, la cual se puede materializar en la búsqueda de soluciones a problemáticas, fenómenos cotidianos o necesidades asociadas con casos presentes en los sistemas productivos, logísticos, de servicios, entre otros.

Según García Hidalgo y Díez Rodríguez, existen diferentes maneras en que los estudiantes aprenden. En términos generales, se basan en las interacciones con lo que observan, escuchan o pueden llegar a intuir (reflexión), a partir de lo cual se forman una idea de la situación (contextualización), es decir que simplemente utilizan la lógica o el sentido común. Por otra parte, la configuración tan variada de las maneras en que los estudiantes aprenden suponen que las estrategias, métodos y didáctica se deben enmarcar en una dinámica de constante evolución (García y Diez, 2018) para de este modo consolidar una estructura de formación integral de los estudiantes en la que se establezca un pensamiento crítico, acompañado por una dosis de creatividad para asumir, representar, analizar, comprender y exteriorizar la esencia de los fenómenos que se han llevado a las aulas como insumo para interactuar con los componentes de un modelo de simulación.

Lo anterior permite adoptar métodos cuya evolución permanente reside en la estructura de pensamiento, pues el estudiante de Ingeniería Industrial debe dar cuenta de los pasos que sigue para llegar a una solución sustentable. Así mismo, debe tomar la decisión bajo un marco de concepción de ideas, búsqueda, experimentación, verificación y mejoramiento permanente. Esta escala de desarrollo del modelamiento y la simulación se convierte en una herramienta para incentivar el pensamiento investigativo y propiciar la innovación.

\section{Metodología}

La construcción del paso a paso de los criterios con los cuales se establecieron las fuentes de información y la diferencia con otros procesos de enseñanza y aprendizaje de la Ingeniería Industrial son producto del análisis de los componentes que integran los modelos clásicos de educación, en comparación con los modelos constructivistas. En tal sentido, se elabora una matriz comparativa basada en los criterios de algunos autores reconocidos de la pedagogía y su aporte frente al uso de la lúdica (tabla 1). Cabe mencionar que todo se valora frente a la conceptualización propia de los procesos de enseñanza y aprendizaje de la ingeniería y se hace una diferenciación en cuanto a los componentes prácticos y las herramientas didácticas que se encuentran a disposición en el laboratorio integral de ingeniería industrial o Lab3i. 
Tabla 1.

Aportes relacionados con la lúdica y categorías de modelos de enseñanza y aprendizaje

\begin{tabular}{ll}
\hline $\begin{array}{l}\text { Aporte relacionado con la } \\
\text { Lúdica/Categoría de modelo }\end{array}$ & Autores \\
\hline $\begin{array}{l}\text { Los ecosistemas académicos } \\
\text { mediados por las tecnologías }\end{array}$ & $\begin{array}{l}\text { González Fernández y } \\
\text { Huerta Gaytán }\end{array}$ \\
\hline Modelo didáctico tradicional & Garrido, Soto y Martínez \\
\hline $\begin{array}{l}\text { Modelo didáctico espontaneís- } \\
\text { ta-activista }\end{array}$ & Delord, Porlán y Harres \\
\hline Modelos didácticos & Aretio García \\
\hline $\begin{array}{l}\text { La lúdica como herramienta } \\
\text { pedagógica }\end{array}$ & Builes, Sánchez y Pinzón \\
\hline $\begin{array}{l}\text { La gama completa de activida- } \\
\text { des de aprendizaje deseados: } \\
\text { terminología, análisis de } \\
\text { fórmulas para su aplicación, } \\
\text { formular nuevos ejemplos }\end{array}$ & Biggs y Tang \\
\hline $\begin{array}{l}\text { Aprendizaje significativo y } \\
\text { colaborativo }\end{array}$ & Anijovich y Mora \\
\hline $\begin{array}{l}\text { Sujeto como el artífice de su } \\
\text { conocimiento }\end{array}$ & Steffe y Gale \\
\hline
\end{tabular}

Fuente: elaboración propia

La meta que se busca con el análisis comparativo de la evolución de los procesos de enseñanza y aprendizaje de la Ingeniería Industrial es establecer un medio para que, con el uso de modelos mentales cuya característica es la interpretación interna de una situación o fenómeno, el estudiante logre construir una visión del contexto real (su cotidianidad) desde una perspectiva a escala. Es decir que con el uso del modelamiento logre concebir un concepto holístico de los componentes y variables que puede encontrar en un escenario industrial existente. En tal sentido, la simulación provee al estudiante de una motivación (espíritu inquisitivo) que le orienta desde su concepción interna de la situación o fenómeno y le ofrece así una perspectiva); le reta a buscar alternativas, fuentes y externalizarlas en un modelo de simulación, que, luego de ser evaluado con el uso de experiencias o por medio de experimentos dentro del laboratorio -0 simplemente se enlace el material didáctico con el software de simulación disponible- le permita establecer un mejor control de las respuestas obtenidas y tomar decisiones objetivas y sustentables en el marco de la gama de potenciales soluciones que se pueden obtener con los modelos de simulación.

Por lo tanto, el proceso de aprendizaje es una interacción entre el nuevo conocimiento que se supone el estudiante debe dominar (interiorizar), el modelo mental en construcción y la realidad en la que el estudiante necesita hacer uso del nuevo conocimiento (Engeström, 2004).

En cuanto a la metodología propuesta para el desarrollo del proyecto de investigación que se asocia con este artículo, se utilizó una conceptualización metodológica inductivadeductiva, dado que parte de la observación (fenómeno, problema) y la experimentación (modelado y simulación) para combinarlas con la identificación de los principios generales (selección de información, análisis de alternativas y resultados) para llegar a la conclusión (toma de decisiones y resolución de conflictos).

\section{Revisión analítica}

Un problema en el contexto de una comunidad, empresa u organización puede parecer una situación que se limita a la intervención del gobierno, la gerencia 0 las políticas (control) que se establecen en algunos casos de modelamiento y simulación para su solución. Sin embargo, dadas sus condiciones particulares y los escenarios que se pueden presentar al realizar una intervención o inmersión con la participación de estudiantes pueden sugerir una o varias incógnitas sobre cómo abordar su desarrollo. Esto lleva a un análisis más profundo de las causas, las cuales incluyen la interacción de los actores, sus diferentes puntos de vista, así como la identificación de los criterios que direccionan los métodos empleados para la 
caracterización de las causas y los efectos que genera dicha problemática.

Bajo esta perspectiva, el estudiante de Ingeniería Industrial puede asumir como elemento vinculante, pero a la vez crítico, entre los requerimientos de la comunidad y las restricciones propias de la problemática. Por ello es necesario que dentro de las prácticas de enseñanza y aprendizaje (PEA) se incluya la visión ABP. Esta mirada configura en el estudiante la capacidad de desarrollar un proceso de aprendizaje basado en problemas (figura 1).

\section{Figura 1.}

Visión de $A B P$ dentro de las prácticas de enseñanza y aprendizaje PEA

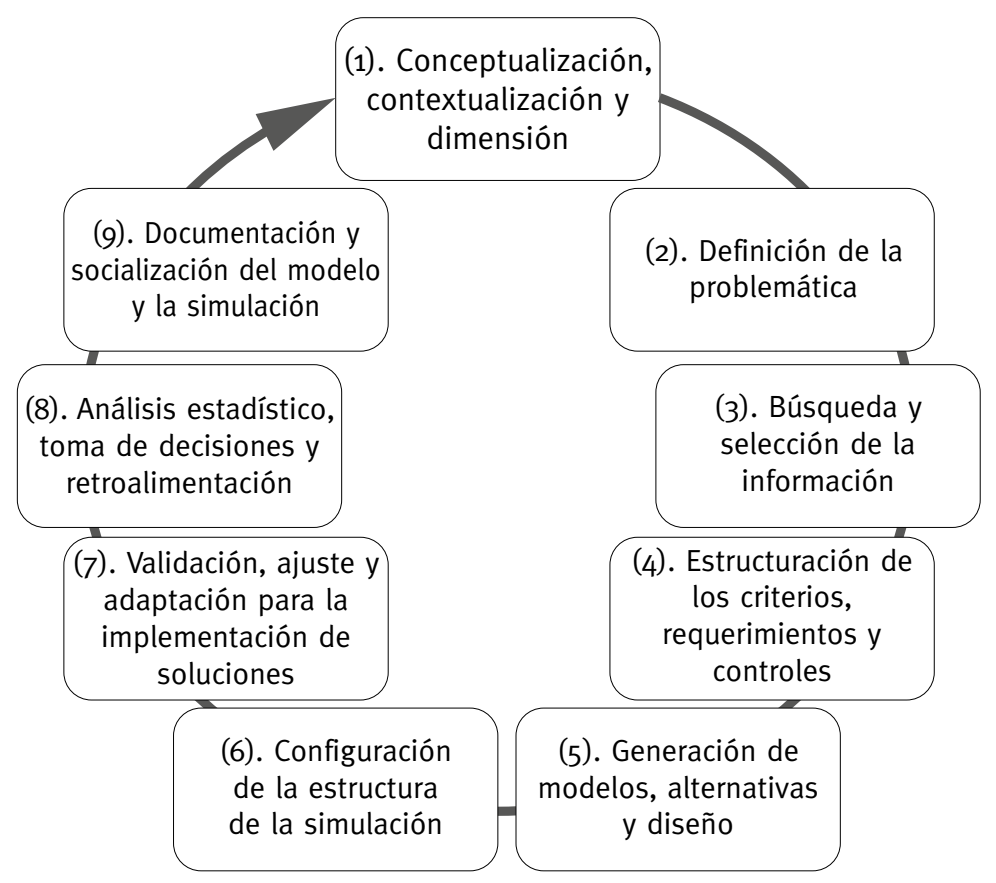

Fuente: elaboración propia

En los primeros semestres los estudiantes de Ingeniería Industrial han de ser orientados al logro de competencias y habilidades que les permitan configurar la concepción (ideación del modelo mental) de un problema, el cual bien puede estar asociado a su cotidianidad dado que el nivel básico requiere una problematización no tan compleja para lograr el aprendizaje significativo, en el que se reconocen las bases y componentes de una conceptualización. Con ello, se logra la contextualización y se identifica la dimensión de la problemática. Este es el primer elemento dentro de la estructura que conforman los otros nueve (9) elementos descritos (figura 1).
La definición de la problemática, responde a la construcción de un propósito en el cual el estudiante de ingeniería debe reflexionar sobre los componentes de un aprendizaje basado en problemas que se integran con el propósito u objetivo de concebir un modelo para simulación. Esto incluye también un proceso de búsqueda y selección de la información para consolidar una estructura ordenada con criterios que fundamentarán las condiciones de obligatorio cumplimiento (aspectos no negociables) como, por ejemplo, los principios físicos, la lógica y/o la secuencia de componentes críticos. Así mismo, se debe pensar cómo se consolidarán los requerimientos que tienen que ver con 
el uso, las restricciones y alternativas que tendrá el modelo para hacer la transferencia a uno o varios diseños pensados en soluciones que deben permitir configurar un arquetipo o estructura de simulación (Ruiz et al., 2018) (figura 2).

Figura 2.

Desagregación de componentes asociados a la visión $A B P$
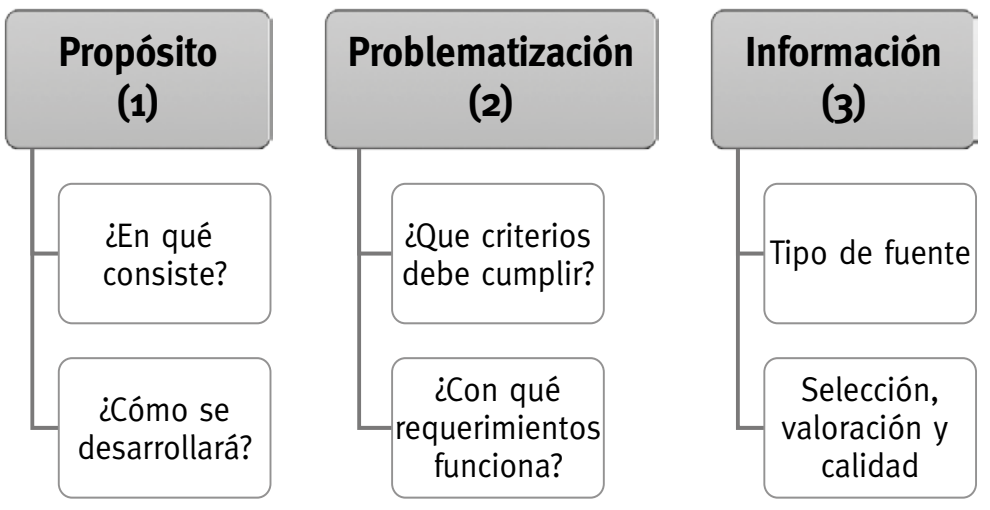
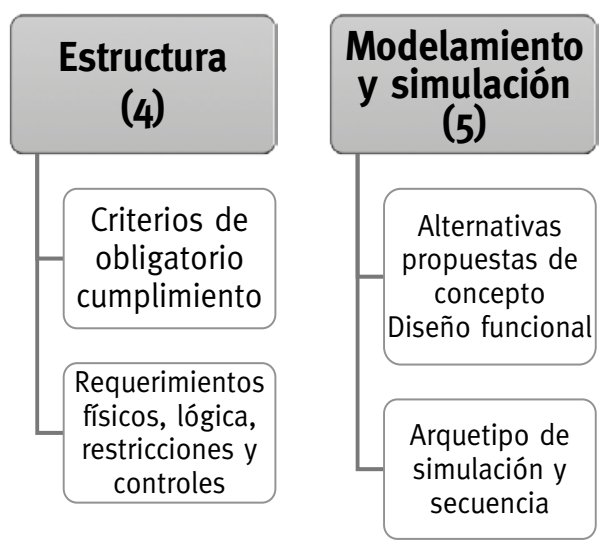

Fuente: elaboración propia

En cuanto al uso de la lúdica para la estrategia de enseñanza y aprendizaje de la Ingeniería Industrial es importante diferenciar lo que se propone en este documento del modo en que se interpreta dicha práctica desde la concepción de las ciencias de la educación. En estas últimas se asocia a actividades con reglas definidas que permiten la integración de roles con carácter simbólico, que incluyen el uso de la imaginación, y que disminuyen conforme se avanza en edad. (Piaget, 2019). Por ello, la propuesta que se desarrolla en el proyecto de investigación en curso denominado "Diseño de lúdicas como estrategia pedagógica en el aprendizaje de Ingeniería Industrial en el laboratorio integral de ingeniería industrial, labzi de Unicatólica (lúdicas labzi)" responde a una dinámica para materializar el logro de la implementación de herramientas didácticas del aprendizaje activo con enfoque lúdico y aplicación de conocimientos adquiridos a lo largo de la carrera, en un contexto práctico que vincula lo académico e investigativo con el contexto empresarial industrial.
Con esto se busca contribuir al mejoramiento del perfil del egresado y por ende, al posicionamiento del programa a nivel regional, inicialmente. Por otra parte, el estudiante de Unicatólica se beneficia con el uso de estas estrategias pedagógicas en la medida en que puede concebir alternativas de solución a fenómenos y problemáticas propias de contextos industriales o de servicios cuya abstracción -a través del aprendizaje activo- permite el diseño de modelos o representaciones de la realidad con un nivel de detalle amplificado que le otorga visión para definir criterios de factibilidad de los procesos orientados a la manufactura, la cadena de suministro y los servicios.

Por otra parte, la interacción dinámica con los modelos permite la experimentación controlada de escenarios a escala. Con ellos pueden efectuar pruebas ensayos y determinar si se requieren ajustes antes de finalizar con la puesta en operación de las oportunidades o alternativas viables para resolver la problemática que se encuentren soportadas en decisiones objetivas, analíticas y validadas con los criterios de responsabilidad y sustentabilidad. 


\section{Estado actual de la investigación y diferencias con otras estrategias didácticas}

El proyecto de investigación se enmarcó en la caracterización de la situación actual del programa de Ingeniería Industrial de Unicatólica. Así mismo, en la adopción de la lúdica como estrategia de enseñanza y aprendizaje. Esta etapa del proyecto permitió establecer criterios orientadores para la estrategia de enseñanza y aprendizaje.

En segundo término se establecieron los criterios funcionales que debería cumplir la infraestructura física disponible para las prácticas de laboratorio en el Lab3l. Para ello se contrastaron y articularon los requerimientos de uso y manejo con los elementos propios de la caracterización que se elaboró del programa de Ingeniería
Industrial. También se analizaron los criterios funcionales asociados con la infraestructura.

Como cierre de la investigación y con base en los resultados, se desarrolló el planteamiento de las estrategias cuyo despliegue será ampliado con mayor detalle en el libro producto de esta investigación. En tal sentido, se hace una mención tangencial de estas en los apartados subsiguientes. Por otra parte, se identificó que la estrategia más ajustada al modelo de enseñanza y aprendizaje de la Ingeniería Industrial se orienta al uso de ABP, el modelado y la simulación.

Las diferencias con otras estrategias se establecen en un esquema que proponen Ausubel, Novak y Hanesian en 1983. Con el cual se aclara por qué el ABP configura una estrategia orientada al aprendizaje significativo. (figura 3).

\section{Figura 3.}

Evolución de los esquemas de enseñanza en ingeniería
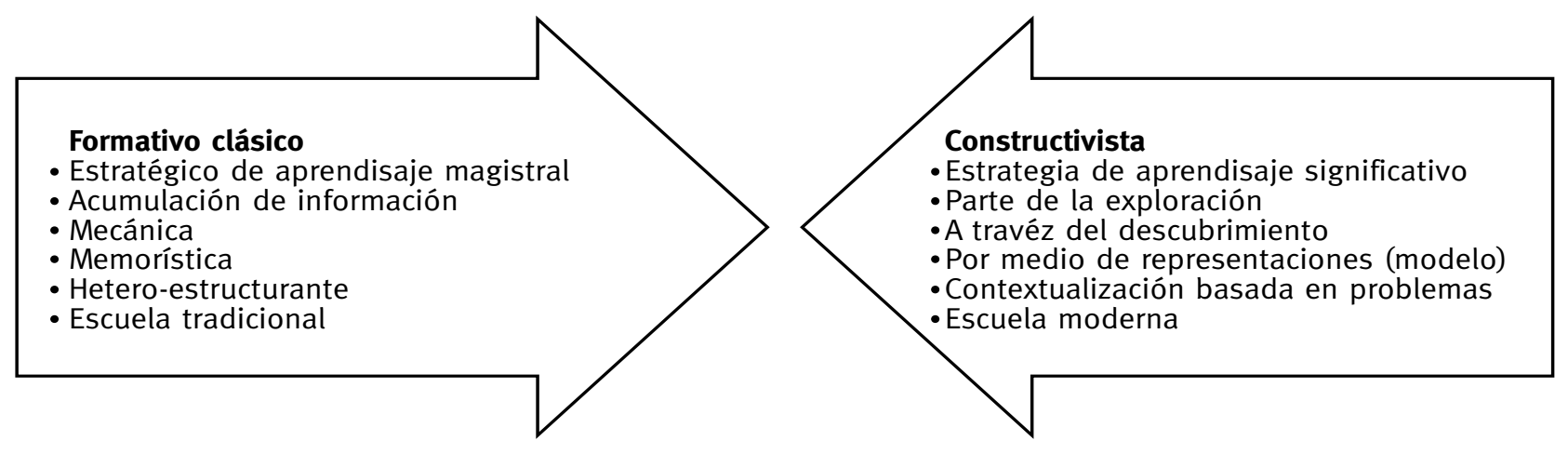

Fuente: adaptado de Ausubel, Novak y Hanesian (1983)

Por otra parte, la configuración de aprendizaje significativo desde el enfoque ABP se alinea con la estructura de competencias genéricas, específicas y las dimensiones que se relacionan con el currículo del programa de Ingeniería Industrial de Unicatólica (tabla 2). 


\section{Tabla 2.}

Dimensiones curriculares asociadas al programa de Ingeniería Industrial

\begin{tabular}{ll} 
& Diseño curricular: \\
& - Fundamentación del programa académico \\
& - Elaboración del perfil profesional del ingeniero de Unicatólica \\
& - Elaboración del plan de estudios \\
& - Elaboración de cursos del plan de estudios \\
\hline Dimensiones del currículo & Desarrollo curricular: \\
& - Acuerdos de formación de calidad para la comunidad académica. \\
& - Mejora de planes de estudio (facilitar la actualización) \\
- Desarrollo de modalidades de formación & - Incorporación de experiencias de innovación \\
- Seguimiento y monitoreo del currículo
\end{tabular}

Fuente: adaptado de Knight, P. (2001) y Barriga et al. (2007)

En la tabla se incluyen criterios asociados al diseño curricular y el desarrollo curricular que se alinean con los propósitos del ABP, particularmente en los componentes de fundamentación del programa académico, elaboración del perfil profesional del ingeniero de Unicatólica, desarrollo de modalidades de formación, incorporación de experiencias de innovación, seguimiento y monitoreo del currículo (tabla 3).

\section{Tabla 3.}

Criterios para el diseño y la reforma curricular de la Facultad de Ingeniería

\begin{tabular}{|c|c|c|}
\hline \multicolumn{3}{|c|}{ Definiciones y conceptos del proyecto de diseño y reforma curricular Facultad de Ingeniería Unicatólica } \\
\hline 1 & $\begin{array}{l}\text { Requerimientos del } \\
\text { entorno }\end{array}$ & $\begin{array}{l}\text { Se plantean para la construcción del perfil profesional del ingeniero egresado } \\
\text { de Unicatólica. }\end{array}$ \\
\hline 2 & $\begin{array}{l}\text { Enfoque por } \\
\text { competencias }\end{array}$ & $\begin{array}{l}\text { "Desarrollo de las capacidades complejas que le permiten a los estudiantes } \\
\text { pensar y actuar en diferentes contextos y/o ámbitos para adquirir } \\
\text { conocimiento a través de la acción” (Álvarez, 2004) }\end{array}$ \\
\hline \multirow[t]{2}{*}{3} & $\begin{array}{l}\text { Gestión de } \\
\text { conocimiento }\end{array}$ & $\begin{array}{l}\text { Se fundamenta en tres métodos: } \\
\text { a. Cognitivo: en este el conocimiento es una entidad fija y representable } \\
\text { (recopilada en bases de datos, archivos y manuales), es fácilmente } \\
\text { compartido. } \\
\text { b. Conexionista: en él el conocimiento reside en las conexiones con } \\
\text { expertos y se orienta a la resolución de problemas. Este (conocimiento) } \\
\text { depende de su relacionamiento con una red para ser compartido. }\end{array}$ \\
\hline & & $\begin{array}{l}\text { c. Constructivista: aquí el conocimiento reside en la mente, el cuerpo y } \\
\text { el sistema social, depende del observador y del pasado. No se comparte } \\
\text { directamente, solo indirectamente a través del diálogo y el pensamiento } \\
\text { analítico. }\end{array}$ \\
\hline 3.1 & $\begin{array}{l}\text { Clasificación de } \\
\text { la gestión del } \\
\text { conocimiento }\end{array}$ & $\begin{array}{l}\text { Se puede agrupar en tres componentes: } \\
\text { a. Gestión de información } \\
\text { b. Capital intelectual } \\
\text { c. Producción, codificación y trasferencia de conocimiento }\end{array}$ \\
\hline
\end{tabular}

Fuente: adaptada de Galindo (1998), Davenport y Prusak (1999) y Valhondo (2003)

\section{La selección de la estrategia}

Como se mencionó, en el estado actual de la investigación la selección de una estrategia de enseñanza y aprendizaje para la ingeniería industrial que se ajuste más al modelo institucional y al programa de Ingeniería Industrial de Unicatólica reúne tres componentes principales. El primero es el aprendizaje basado 
en problemas (ABP) en el que el análisis efectuado permite inferir que la estructura de pensamiento del ingeniero industrial en formación parte de la curiosidad frente a una situación que se desea mejorar (esta se considera como un problema).

Por otra parte, dicho problema induce al estudiante a una búsqueda de información, con lo cual amplía su conocimiento sobre la situación problemática. Esto lo conduce a concebir un modelo que está en su mente (modelo mental), el cual se materializa en un modelo o representación de dicha situación. Con esto, el estudiante plantea alternativas de solución y puede analizar las interacciones entre las variables, entidades y demás componentes de un modelo.

No obstante, para comprender su comportamiento el alumno debe efectuar la simulación en la que construye escenarios controlados de la situación y puede llegar a inferir luego del análisis estadístico cuál es la solución al problema que inicialmente se le propuso. Así, la combinación de estos tres componentes se convierte en una estrategia de enseñanza y aprendizaje significativo.

\section{Elementos de modelado y simulación en Ingeniería Industrial}

En el contexto de la Ingeniería Industrial como disciplina la simulación aporta una herramienta avanzada para la toma de decisiones en el sentido en que permite evaluar una problemática desde dos tipos de análisis. Uno asociado al pensamiento sistémico que es la dinámica de sistemas y otro más cercano a la interpretación de eventos discretos (Loaiza et al., 2019).

En el caso de la Ingeniería Industrial la simulación se asocia con el análisis de eventos discretos al integrar aspectos relevantes de modelamiento y simulación frente a fenómenos cotidianos, lo que permite la observación, análisis y comprensión de las problemáticas que parte de lo simple hasta estructurar un modelo complejo de la realidad. Por su parte, la dinámica de sistemas está más orientada a la comprensión de las interrelaciones del sistema con sus componentes desde una concepción más de pensamiento sistémico.

La estructura de pasos de modelado y simulación se puede resumir en una problemática que se plantea a los estudiantes mediante la presentación de un caso que contiene una situación propia de los escenarios industriales, en los cuales se establecen de manera general, los elementos de información y de referencia que servirán de datos de entrada para establecer los pasos que se mencionan en la (figura 2). Pero en esta etapa del desarrollo de la práctica del modelamiento y la simulación se deben incluir los siguientes pasos que se describen en la (figura 4). Todos los aspectos que aquí se mencionan se ajustan al decreto 3963 de octubre de 2009 del Ministerio de Educación Nacional (Estepa, R. 2015) y ajustados según los requerimientos institucionales de Unicatólica.

Figura 4.

Pasos para el desarrollo práctico del modelado y la simulación

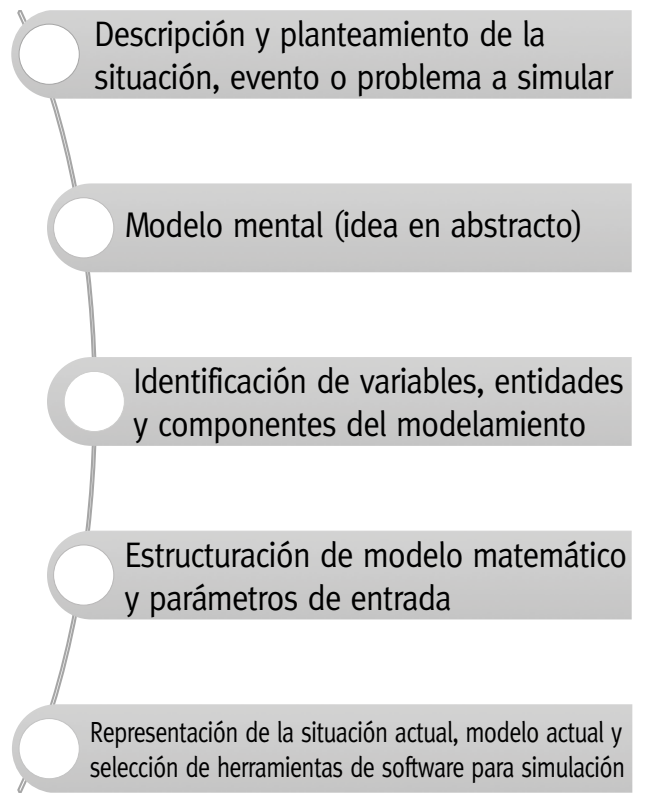

Fuente: elaboración propia 


\section{La simulación como una herramienta de externalización}

La simulación es una representación de la realidad. El uso de los juegos de simulación constituye un variado cúmulo de potentes herramientas de aprendizaje ya que, a diferencia de las situaciones o fenómenos reales, estas se pueden adaptar y controlar sin riesgo para ciertos propósitos de aprendizaje. Aunque la idea de utilizar la simulación como una herramienta pedagógica no es nueva, su aplicación típica se encuentra propiamente en la dinámica de sistemas. En Ingeniería Industrial se comienzan a explorar sus ventajas hacia 1960 en el Massachusetts Institute of Technology (MIT) (Kaminsky y Simchi, 1998). Como resultado se desarrolla el concepto de juegos de empresa.

\section{Los juegos de empresa o juegos de roles de empresa}

Luego de los desarrollos en el uso de la tecnología de software como herramienta de educación en ingeniería y al considerar los resultados obtenidos con el famoso Beer Game, o juego de la cerveza, Miles, Biggs y Schubert clasificaron este tipo de juegos en dos ramas: por un lado, los que implican un análisis completo del sistema empresarial y por el otro, los asociados a procesos de mercadeo, producción, finanzas y talento humano (Miles et al., 1986). En tal sentido, la aplicación del modelado y la simulación permiten una experiencia muy cercana a la realidad cuando el estudiante de Ingeniería Industrial comienza a pensar en la manera de configurar los criterios, enlazar las variables y determinar las entidades que se deben configurar para representar un sistema que se acerque a la situación, fenómeno o problema que se quiere solucionar con la ayuda de la simulación.

\section{El modelamiento y la simulación}

Son dos herramientas avanzadas de ingeniería orientadas a resolver, analizar y validar eventos, situaciones o fenómenos cuyo comportamiento se debe observar para tomar una decisión sustentada en el nivel de confianza máximo. Su núcleo principal es la construcción de representaciones o modelos, los cuales deben cumplir con ciertos criterios para garantizar su éxito.

Por lo cual, su uso en una estrategia para la enseñanza de la Ingeniería Industrial favorece la interpretación que el estudiante realiza de su entorno. Finalmente, la combinación de la lúdica entendida como una actividad de aprendizaje significativo responde a la necesidad de diseñar alternativas, construir modelos y experimentar con los mismos para comprender las interrelaciones de sus variables con los componentes y entidades que componen el sistema o modelo. Por ello, cuando se llevan los componentes fundamentales (estudiante, docente y PEA) al laboratorio se pueden configurar -con la ayuda de material didáctico- modelos a escala de una línea de manufactura, en los que el modelo mental se representa a través del diagrama de flujo del proceso y el modelo matemático corresponde a la interacción de las variables con las entidades del sistema.

\section{Conclusiones y recomendaciones}

Las prácticas de enseñanza y aprendizaje de la Ingeniería Industrial de Unicatólica se deben dirigir dentro de un esquema de interacción dinámico en que el estudiante y el docente puedan construir un modelo mental de la realidad, fenómeno o problema que se busca solucionar. Esto incluye la identificación de variables dentro del sistema o modelo.

La secuencia ordenada de pasos que se requieren para construir un concepto parten 
de lo que internamente el estudiante de Ingeniería Industrial de Unicatólica elaboró en su esquema mental; de allí viene el proceso de externalización que -con la guía del docente- le permitirán considerar los criterios de búsqueda de información, su selección y la validación de los datos con los que alimentará el modelo de simulación.

Con la información de entrada validada, el estudiante de Ingeniería de Unicatólica podrá diseñar un plan, para construir un modelo matemático que contenga las variables, parámetros y entidades a representar en el software o con el material didáctico disponible en el Labzi.

Los experimentos o pruebas se enmarcan en la generación de escenarios guiados por el docente y que el estudiante de Ingeniería utilizará como insumo para el análisis de situaciones y presentar una evaluación gráfica en la que se observe cuál de las simulaciones ofrece el mayor índice de confiabilidad.

\section{Referencias}

Anijovich, R., y Mora, S. (2010). Enseñar en aulas heterogéneas. En N. Anijovich, y S. Mora. Estrategias de enseñanza: otra mirada al quehacer en el aula (pp. 101-105). Aique grupo editor.

Álvarez, R. P. (2004). Formación superior basada en competencias, interdisciplinariedad y trabajo autónomo del estudiante. Revista iberoamericana de educación, 35(1), 1-33.

Aretio, L. G. (2017). Educación a distancia y virtual: calidad, disrupción, aprendizajes adaptativo y móvil. RIED. Revista Iberoamericana de Educación a Distancia, 20(2), 9-25.

Ausubel, N., y Novak, J. D. y Hanesian (1983). Psicología Educativa: Un punto de vista cognoscitivo. Trillas.

Barriga, F., Lule González M. L., Pacheco D., Saad E., y Rojas-Drummond, S. (2007). Metodología de Diseño Curricular para educación Superior. Trillas.
Para la toma de decisiones es importante que se hayan realizado los ajustes al modelo de la situación actual, es decir un modelo mejorado donde se corrigen los vacíos estructurales del modelo de simulación para finalmente presentar un informe que retroalimente su aprendizaje haciéndolo significativo.

Las conclusiones que se presentan en este artículo son parciales debido a las restricciones que plantea un escenario propio de la situación de la pandemia por la enfermedad de COVID-19. Este contexto no permite efectuar las intervenciones presenciales con los estudiantes o pruebas de campo de las estrategias planteadas, y sin ellas no es posible establecer la línea base para coordinar las estrategias con mejores indicadores de resultado. En tal sentido, esto se propone como escenario futuro de desarrollo una vez superada la contingencia, por lo pronto, se buscan alternativas virtuales de implementación para estas intervenciones.

Biggs, J., y Tang, C. (2011). Train-the-Trainers: Implementing Outcomes-Based Teaching and Learning in Malaysian Higher Education. Malaysian Journal of Learning and Instruction, 8, 1-19.

Builes, J. A. J., Sánchez, R. E. A., y Pinzón, L. D. J. (2016). Métodos de búsqueda usando los algoritmos de enjambre de partículas y genético. Lámpsakos, (16), 52-60.

Davenport, T., y Prusak, L. (1999). El Conocimiento activo: Cómo las Organizaciones manejan lo que ellos saben. Prentice Hall.

Delord, G., Porlán, R., y Harres, J. (2017). La importancia de los proyectos y redes innovadoras para el avance de la Enseñanza de las Ciencias: El caso de un profesor de la Red IRES. Revista Eureka sobre Enseñanza y Divulgación de las Ciencias, 653-665.

Engeström, Y. (2004). New forms of learning in co configuration work. Journal of Work- 
place Learning, 16(1/2), 11-21. https://doi. org/10.1108/13665620410521477

Escribano González, A., y Valle López, A. del. (2018). El aprendizaje basado en problemas (ABP): una propuesta metodológica en educación superior. Narcea ediciones.

Estepa, R. P., \& Rodríguez, R. B. (2015). Una mirada a las pruebas saber pro en la calidad de la educación superior en Colombia. Pensamiento Republicano, (3).

Fernández, M. O. G., \& Gaytán, P. H. (2019). Experiencia del aula invertida para promover estudiantes prosumidores del nivel superior. RIED. Revista Iberoamericana de Educación a Distancia, 22(2), 245-263.

Galindo, C. (1998). El currículo implementado como indicador del proceso educativo. Análisis de los Resultados y Metodología de las Pruebas CRECER. Documento de Trabajo, № 13. Ministerio de Educación, programa especial Mejoramiento de la Calidad de la Educación Peruana-MECEP.

García Hidalgo, Y., y Díez Rodríguez, H. (2018). Aprendizaje activo en los estudiantes de ingeniería industrial. Ojeando La Agenda, 51, 1-10.

Garrido, G. V., Soto, I. R. S., \& Martínez, H. A. (2018). Prácticas pedagógicas en ingeniería: un análisis desde los docentes. Revista de Investigación № 95 Vol. 42 Septiembre-Diciembre, 2018, 42(95), 102.

Kaminsky, P., y Simchi, D. (1998). A New Computerized Beer Game: A Tool for Teaching the Value of Integrated Supply Chain Management 1. En Supply Chain and Technology Management. The Production and Operations Management Society
Knight, P. T. (2001). Complexity and curriculum: A process approach to curriculum-making. Teaching in higher education, 6(3), 369-381.

Loaiza, M. E. B., Suta, M. G., Salinas, L. M. O., Ramírez, M. C., y lodice, R. (2019, agosto 31). Herramientas para evaluar las habilidades metacognitivas en estudiantes de ingeniería al resolver problemas en simulación de eventos discretos [ponencia]. Encuentro Internacional de Educación en Ingeniería 2019.

Miles, W. G., Biggs, W. D., y Schubert, J. N. (1986). Student Perceptions of Skill Acquisition through Cases and a General Management Simulation: A Comparison. Simulation \& Games, 17(1), 7-24. https://doi.org/10.1177/0037550086171002

Piaget, J. (2019). La formación del símbolo en el niño: Imitación, juego y sueño. Imagen y representación. Fondo de Cultura Económica.

Ruiz, C. R., Castiblanco, I. A., Cruz, J. P., Pedraza, L. C., y Londoño, D. (2018). Juegos de simulación en la enseñanza de la Ingeniería Industrial: caso de estudio en la Escuela Colombiana de Ingeniería Julio Garavito. Entre Ciencia e Ingeniería, 12(23), 48. https://doi. org/10.31908/19098367.3702

Salamanca, N. M. M. (2017). Modelo teórico-didácticotecnológico didáctico para el aprendizaje de las matemáticas en la formación básica secundaria. Itinerario Educativo, 30(68), 65-77.

Steffe, L. P., \& Gale, J. E. (Eds.). (1995). Constructivism in education. Psychology Press.

Valhondo, D. (2003). Gestión del conocimiento: del mito a la realidad. Ediciones Díaz de Santos. 